Original Research Paper

\title{
Statistical Inferences of the Rest Lifetimes of Ball Bearings' Residual Lifetimes Based on Rayleigh Residual Type II Censored Data
}

\author{
${ }^{1}$ Ghassan K. Abufoudeh, ${ }^{2}$ Omar M. Bdair and ${ }^{1}$ Raed R. Abu Awwad \\ ${ }^{I}$ Department of Basic Sciences, Faculty of Arts and Sciences, University of Petra, Amman, Jordan \\ ${ }^{2}$ Department of Physics and Basic Sciences, Faculty of Engineering Technology, \\ Al Balqa Applied University, Amman 11134, Jordan
}

\author{
Article history \\ Received: 05-04-2016 \\ Revised: 23-06-2016 \\ Accepted: 28-07-2016 \\ Corresponding Author: \\ Omar M. Bdair \\ Department of Physics and \\ Basic Sciences, Faculty of \\ Engineering Technology, Al \\ Balqa Applied University, \\ Amman 11134, Jordan \\ Email: bdairmb@yahoo.com
}

\begin{abstract}
In this study, we consider statistical inference problems for the residual life data that come from the Rayleigh model based on type II censored data. Maximum likelihood and Bayesian approaches are used to estimate the scale and location parameters for the Rayleigh model, the Gibbs sampling procedure is used to draw Markov Chain Monte Carlo (MCMC) samples and MCMC samples have been used to compute the Bayes estimates and to construct symmetric credible intervals. Furthermore, we estimate the posterior predictive density of the future ordered data and then obtain the corresponding predictors. The Gibbs and Metropolis samplers are used to predict the life lengths of the missing lifetimes in multiple stages of the residual type II censored sample. Numerical comparisons for a real life data involving the ball bearings' lifetimes and the artificial data are conducted to assess the performance of the parameters' estimators and the predictors of future ordered data using some specialized computer programs.
\end{abstract}

Keywords: Residual Life Data, Rayleigh Distribution, Type II Censored Data, Maximum Likelihood Estimation, Bayes Estimation, Bayes Prediction, Gibbs Sampling, MCMC Samples

\section{Introduction}

In the last few decades, the prediction problem of future lifetimes, based on a sequence of known lifetimes, had a great reputation among other important sciences. Sometimes, we may ignore some known lifetimes called as non effective, this term (non effective lifetimes) is used to denote lifetimes that are less than some predetermined time $t$. This predetermined time $t$ may be determined as a lifetime that some chipset will survive for a small lifetime even though it has some artificial error. In this study, we introduce an example of some ball bearings lifetimes, where we will ignore the lifetimes which are less than other known $\mathrm{t}$ which is considered by experts to be the lifetime that a single ball bearing will survive with its artificial error. Based on the remaining lifetimes of non defective ball bearings, we will use the methodology that is introduced below to predict the future lifetimes of still surviving ball bearings.
The Rayleigh distribution was first introduced by Lord Rayleigh (1880), it was originally derived in connection with a problem in acoustics and has been used as the distance distribution among individuals in a spatial Poisson process. For more details on the Rayleigh distribution the reader is referred to Johnson et al. (1994). The origin and other aspects of this distribution can be found in Siddiqui (1962) and Miller and Sackrowttz (1967).

The Rayleigh distribution is one of the most widely used distributions in reliability and survival analysis representation of the distribution or survival function. The distribution has been used very effectively for analyzing lifetime data, mainly when the data are censored. This means that the Rayleigh distribution is very common in studying most life testing experiments. For more details about the Rayleigh distribution, one may refer to Dey (2009). Dey et al. (2014) studied different methods of estimation for the two parameters Rayleigh distribution, they proved that the Bayes 
estimator is better than other estimators. They also presented the best priors for the two parameters based on their study of the existence and non existence of conjugate and Jeffreys' priors. More recent Dey et al. (2016) studied the Estimation and Bayes prediction problems using non-informative prior for the Rayleigh distribution under progressively type II censored data.

Suppose $\mathrm{n}$ items are kept under observation until failure, these items could be systems, components or computer chips in reliability study experiments, or they could be patients under certain drug or clinical conditions and their lifetimes $\underset{\sim}{X}=\left(X_{1}, X_{2}, \ldots, X_{n}\right)$ following the Rayleigh distribution with probability density function (pdf):

$$
f(x, \lambda, \mu)=\left\{\begin{array}{cc}
2 \lambda(x-\mu) e^{-\lambda(x-\mu)^{2}} & \text { if } x>\mu, \lambda>0 \\
0 & \text { if } x \leq \mu
\end{array}\right.
$$

and cumulative distribution function (cdf):

$$
F(x, \lambda, \mu)=1-e^{-\lambda(x-\mu)^{2}}, x>\mu, \lambda>0
$$

The scale and the location parameters are $\lambda>0$ and $\mu>0$.

For a reason or another, one may terminate the experiment at the $r$-th failure, $X_{r: n}$, so we obtain a type II censored sample. Here $r$ is fixed and $X_{r: n}$, the duration of the experiment is random. The likelihood function in this case is:

$$
\begin{aligned}
& L(\lambda, \mu \mid \underset{\sim}{X})=\frac{n !}{(n-r) !} \prod_{i=1}^{r} f\left(x_{i} \mid \lambda, \mu\right)\left[1-F\left(x_{r} ; \theta\right)\right]^{n-r}, \\
& x_{1}, x_{2}<\ldots<x_{r}
\end{aligned}
$$

Let $X$ be a non-negative random variable, in many real life situations the random variable:

$$
Y=X-t \mid X>t, t>0
$$

is denoted to the residual life random variable which is greater than a predetermined age $t$. These residual life data and their ordering can be effectively applied in reliability theory and survival analysis, they also plays an important role if one observes the residual lifetimes only. This type of data arise naturally in survival actuarial studies.

Now, assume we have complete data for the residual lifetimes $Y_{1}, Y_{2}, \ldots, Y_{n}$, where $Y_{i}=X_{i}-t \mid X_{i}>t, i=1,2, \ldots, n$ are the residual lifetimes random variables after deleting the non effective lifetimes. The cdf of the residual lifetimes variable can be calculated as:

$$
G(y)=\operatorname{Pr}(X-t-<y \mid X>t)=\frac{F(t+y)-F(t)}{1-F(t)}, y>0
$$

and the corresponding pdf is given by:

$$
g(y)=\frac{f(t+y)}{1-F(t)}, y>0
$$

Taking into consideration the Rayleigh distribution, this leads to:

$$
G(y)=1-e^{-\lambda\left[(t+y-\mu)^{2}-(t-\mu)^{2}\right]}, y>0
$$

and:

$$
g(y)=2 \lambda(t+y-\mu) e^{-\lambda\left[(t+y-\mu)^{2}-(t-\mu)^{2}\right]}, y>0
$$

This paper discusses two points; First the computing of the MLEs and Bayes estimates of $\mu$ and $\lambda$ under squared error loss function then comparing their performances using extensive computer simulations. Second considering the prediction of future order statistics based on type-II censored observations. Prediction of unobserved data is important especially in actuarial, medical and engineering sciences. Balakrishnan and Rao (1997) and Kaminsky and Nelson (1998).

The rest of this paper is prepared as follows: In section 2, we estimate the MLEs for both the scale and the location parameters of the Rayleigh distribution. The Bayes estimates for the scale and the location parameters $\lambda$ and $\mu$, respectively, are derived in section 3 . In section 4 , we introduce the Bayes prediction problem of the unknown data from the future sample. Numerical simulations and data analysis illustrating the obtained results, depending on both artificial and real life data, are included in section 5. Theoretical and numerical results' conclusion is introduced in section 6 .

\section{Maximum Likelihood Estimation}

In this section, we derive the Maximum Likelihood Estimators (MLEs) of the parameters $\lambda$ and $\mu$ from the Rayleigh model based on type II censored data using the residual life observations. Let $Y_{1: n}<Y_{2: n}<\ldots<Y_{r: n}$ be a type II censored sample of size $r(1<r<n)$, for notations simplicity we will refer $y_{i}$ for $Y_{i: n}$. Using Equation 3, 6 and 7, the likelihood function is given by:

$$
\begin{aligned}
& L\left(\lambda, \mu \mid y_{1}, \ldots, y_{r}\right)=\frac{n !}{(n-r) !} 2^{r} \lambda^{r} \prod_{i=1}^{r}\left(t+y_{i}-\mu\right) \\
& e^{-\lambda \sum_{i=1}^{r}\left[\left(t+y_{i}-\mu\right)^{2}-(t-\mu)^{2}\right]} e^{-\lambda(n-r)\left[\left(t+y_{r}-\mu\right)^{2}-(t-\mu)^{2}\right]}
\end{aligned}
$$

Differentiating the natural logarithm of the likelihood function with respect to $\lambda$ and $\mu$ and equating the resulting terms to zero, we obtain: 


$$
\begin{aligned}
& \sum_{i=1}^{r} \frac{-1}{\left(t+y_{i}-\mu\right)} \\
& +\frac{2 r \sum_{i=1}^{r} y_{i}+2 r(n-r) y_{r}}{\sum_{i=1}^{r}\left[y_{i}^{2}+2 y_{i}(t-\mu)\right]+(n-r)\left[y_{r}^{2}+2 y_{r}(t-\mu)\right]}=0
\end{aligned}
$$

and:

$$
\lambda=\frac{r}{\sum_{i=1}^{r}\left[y_{i}^{2}+2 y_{i}(t-\mu)\right]+(n-r)\left[y_{r}^{2}+2 y_{r}(t-\mu)\right]}
$$

Once the MLE of $\mu$, say $\hat{\mu}$, is obtained and computed as a numerical solution of Equation 8, the MLE of $\lambda$, say $\hat{\lambda}$, can be obtained directly from Equation 9. For more details about the existence and uniqueness of these MLEs, Balakrishnan and Kateri (2008).

\section{Bayes Estimate}

For the Bayesian inference and life testing plan, we need to assume some prior distributions to obtain the Bayes Estimates (BEs) of $\lambda$ and $\mu$ and the corresponding credible intervals based on type II censored data. When the location parameter $\mu$ is known, the natural choice of the scale parameter $\lambda$ has a conjugate gamma prior $\operatorname{Gamma}(a, b)$, with pdf:

$$
\pi_{1}(\lambda \mid a, b)=\left\{\begin{array}{cc}
\frac{b^{a}}{\Gamma(a)} \lambda^{a-1} e^{-b \lambda} & \text { if } \lambda>0 \\
0 & \text { if } \lambda \leq 0
\end{array}\right.
$$

with the hyper-parameters $a>0, b>0$ and $\Gamma(a)$ $=\int_{0}^{\infty} x^{a-1} e^{-x} d x$.

When the location parameter $\mu$ is unknown, the continuous conjugate priors do not exist and have no specific form, Kaminskiy and Krivtsov (2005) and Dey (2014). Squared Error Loss (SEL) function is assumed to compute the Bayes estimates. We assume the prior of $\mu, \pi_{2}(\mu)$ with support $(0, \infty)$, for more details Kundu (2008).

\section{Location Parameter Known}

Based on a Type II censored data $Y_{1: n}<Y_{2: n}<\ldots<$ $Y_{r: n} ;(1 \leq r \leq n)$ and the priors of $\lambda$ and $\mu$, we obtain the joint posterior of $\lambda$ and $\mu$ as:

$$
\begin{aligned}
& \pi\left(\lambda, \mu \mid y_{1}, \ldots, y_{r}\right) \propto \lambda^{r+a-1} \prod_{i=1}^{r}\left(t+y_{i}-\mu\right) \\
& \left.e^{-\lambda\left(b+\sum_{i=1}^{r}\left[\left(t+y_{i}-\mu\right)^{2}-(t-\mu)^{2}\right]\right.}\right] e^{-\lambda(n-r)\left[\left(t+y_{r}-\mu\right)^{2}-(t-\mu)^{2}\right]} \pi_{2}(\mu)
\end{aligned}
$$

The conditional posterior distribution of $\lambda$ given $\mu$ and $y_{1}, \ldots, y_{r}$ is given by:

$$
\pi_{1}\left(\lambda \mid \mu, y_{1}, \ldots, y_{r}\right) \propto \lambda^{r+a-1} e^{-\lambda\left(\begin{array}{l}
b+\sum_{i=1}^{n}\left[\left(t+y_{i}-\mu\right)^{2}-(t-\mu)^{2}\right] \\
+(n-r)\left[\left(t+y_{r}-\mu\right)^{2}-(t-\mu)^{2}\right]
\end{array}\right)}
$$

which is:

$\operatorname{Gamma}\left(\begin{array}{l}r+a, b+\sum_{i=1}^{r}\left[\left(t+y_{i}-\mu\right)^{2}-(t-\mu)^{2}\right] \\ +(n-r)\left[\left(t+y_{r}-\mu\right)^{2}-(t-\mu)^{2}\right]\end{array}\right)$

Therefore, the $\mathrm{BE}$ of $\lambda$, say $\tilde{\lambda}_{\text {Bayes }}$, is readily concluded to be:

$$
=\frac{\tilde{\lambda}_{\text {Bnges }}}{b+\sum_{i=1}^{r}\left[\left(t+y_{i}-\mu\right)^{2}-(t-\mu)^{2}\right]+(n-r)\left[\left(t+y_{r}-\mu\right)^{2}-(t-\mu)^{2}\right]}
$$

Also, the (1- $\beta) 100 \%$ Bayesian credible interval for $\lambda$ is given by $\left(\lambda_{L}, \lambda_{U}\right)$ where:

$$
\int_{\lambda_{L}}^{\lambda_{U}} \pi_{1}\left(\lambda \mid \mu, y_{1}, \ldots, y_{r}\right) d \lambda=1-\beta
$$

So:

$\operatorname{Pr}\left(\lambda_{L}<\lambda<\infty\right)=1-\frac{\beta}{2}$ and $\operatorname{Pr}\left(\lambda_{U}<\lambda<\infty\right)=\frac{\beta}{2}$

Using Equation 11, 13 and the incomplete gamma function which is defined as $\Gamma(a, c)=$ $\int_{c}^{\infty} x^{a-1} e^{-x} d x, a>0, c>0$, we obtain:

$\Gamma\left(r+a, u \lambda_{L}\right)=\left(1-\frac{\beta}{2}\right) \Gamma(r+a)$

and:

$$
\Gamma\left(r+a, u \lambda_{U}\right)=\frac{\beta}{2} \Gamma(r+a)
$$

where:

$$
\begin{aligned}
& u=b+\sum_{i=1}^{r}\left[\left(t+y_{i}-\mu\right)^{2}-(t-\mu)^{2}\right] \\
& +(n-r)\left[\left(t+y_{r}-\mu\right)^{2}-(t-\mu)^{2}\right]
\end{aligned}
$$


Now, using a suitable numerical method to solve Equation 14 and 15, we obtain $\left(\lambda_{L}, \lambda_{U}\right)$.

\section{Location Parameter Unknown}

Here, we explain how to obtain the BEs and the corresponding credible intervals for $\lambda$ and $\mu$ when both parameters are unknown. The conditional posterior distribution of $\mu$ given $\lambda$ and $y_{1}, \ldots, y_{r}$ is given by:

$\pi_{2}\left(\mu \mid \lambda, y_{1}, \ldots, y_{r}\right) \propto \pi_{2}(\mu) \prod_{i=1}^{r}\left(t+y_{i}-\mu\right) \frac{1}{u^{r+a}}$

The BE of any function of $\lambda$ and $\mu($ say $g(\lambda, \mu))$ is:

$$
\begin{aligned}
& \tilde{g}(\lambda, \mu)=E_{\lambda, \mu \mid y_{1}, \ldots, y_{r}}(g(\lambda, \mu)) \\
& =\int_{0}^{\infty} \int_{0}^{\infty} g(\lambda, \mu) \pi\left(\lambda, \mu \mid y_{1}, \ldots y_{r}\right) d \lambda d \mu
\end{aligned}
$$

Since the expectation in Equation 17 may not be obtained in an explicit form, we suggest to use Gibbs sampling technique to generate samples from the conditional posterior distribution of $\lambda$ and $\mu$. The generated samples are used to compute BEs of $\lambda$ and $\mu$ and also to construct credible intervals of $\lambda$ and $\mu$. The MCMC samples $\left(\lambda_{i}, \mu_{i}\right) ; i=1,2, \ldots M$ are generated using the following algorithm:

Step 1: Generate $\mu$ from $\pi_{2}\left(\mu \mid \lambda, y_{1}, \ldots, y_{r}\right)$ using the Metropolis-Hasting algorithm.

Step 2: For each $\mu$, generate $\lambda$ from $\pi_{1}(\lambda \mid \mu$, data).

Step 3: Repeat steps 1 and $2 M$ times to obtain MC samples $\left\{\left(\lambda_{i}, \mu_{i}\right) ; i=1,2, \ldots M\right\}$.

The resulting samples $\left(\lambda_{i}, \mu_{i}\right)$ are used to approximate the BEs and to construct the credible intervals for the parameters.

\section{Bayes Prediction}

An important feature in Bayes analysis is the Bayes prediction of future data based on the current available sample which is also known as informative sample. We mainly consider the estimation of the posterior predictive density of the future data based on the current observed data. Our objective is to provide the prediction for the future data of an experiment depending on the results obtained from an informative experiment. Here we suggest the following method, we call it posterior density based method:

Let $y_{1}<y_{2}<\ldots<y_{r}$ be the observed sample (informative sample) and $y_{r+1}<y_{r+2}<\ldots<y_{n}$ be the unobserved future sample, our goal is to predict $y_{s}, r<s$ $\leq n$. The posterior predictive density of $y_{s}$ given the observed data $\underset{\sim}{y}=\left(y_{1}, y_{2}, \ldots, y_{r}\right)$ is defined as:

$$
\begin{aligned}
& \pi_{y_{s}}\left(y \mid y_{1}, \ldots, y_{r}\right) \\
& =\int_{0}^{\infty} \int_{0}^{\infty} g_{y_{s} \mid y_{1}, \ldots, y_{r}}(y \mid \lambda, \mu) \pi\left(\lambda, \mu \mid y_{1}, \ldots, y_{r}\right) d \lambda d \mu, y_{s}>y_{r}
\end{aligned}
$$

where, $g_{y_{s} \mid y_{1}, \ldots, y_{r}}(y \mid \lambda, \mu)$ is the conditional density of $y_{s}$ given $\lambda, \mu$ and data $\underset{\sim}{Y}$, see for example Chen et al. (2000).

Using the Markovian property of the conditional order statistics, David and Nagaraja (2003), the conditional pdf of $y_{s}$ given $\underset{\sim}{Y}$ is just the conditional pdf of $y_{s}$ given $y_{r},(r+1 \leq s \leq n)$. Specifically:

$$
\begin{aligned}
& g_{y_{s} \mid y_{1}, \ldots, y_{r}}\left(y_{s} \mid \lambda, \mu\right)=g_{y_{s} \mid y_{r}}\left(y_{s} \mid \lambda, \mu\right)=\frac{g_{r ; s}\left(y_{r}, y_{s}\right)}{g_{r}\left(y_{r}\right)} \\
& =c\left[1-e^{-\lambda\left[(t+y-\mu)^{2}-(t-\mu)^{2}\right]}\right]^{s-r-1} e^{-\lambda(n-s+1)\left[(t+y-\mu)^{2}-\left(t+y_{r}-\mu\right)^{2}\right]} 2 \lambda(t+y-\mu)
\end{aligned}
$$

where, $g_{r ; s}\left(y_{r}, y_{s}\right)$ is the joint pdf of the $r$-th and $s$-th order statistics from a sample of size $\mathrm{n}$ from the parent distribution $G($.$) . One can observe that the conditional$ density of $y_{s}$ given $y_{r}$ is just the marginal density of ( $s$ $r)$-th order statistics from a sample of size $(n-r)$ from the left truncated distribution of $G($.$) at y_{r}$. Using the binomial expansion, we conclude:

$$
\begin{aligned}
& g_{y_{s} \mid y_{1}, \ldots, y_{r}}(y \mid \lambda, \mu) \\
& =2 c \lambda \sum_{i=0}^{s-r-1}\left(\begin{array}{c}
s-r-1 \\
i
\end{array}\right)(-1)^{i} e^{-(n-s+i+1) \lambda\left[(t+y-\mu)^{2}-\left(t+y_{r}-\mu\right)^{2}\right]}(t+y-\mu)
\end{aligned}
$$

where, $c=\frac{(n-r) !}{(s-r-1) !(n-s) !}$. So, the posterior predictive density of $Y_{s: n}$ at any point $y>y_{r}$ can be written as:

$$
\begin{aligned}
& \pi_{y_{s}}\left(y \mid y_{1}, \ldots, y_{r}\right) \\
& =2 c \int_{0}^{\infty} \int_{0}^{\infty} \lambda \sum_{i=0}^{s-r-1}\left(\begin{array}{c}
s-r-1 \\
i
\end{array}\right)(-1)^{i} e^{-(n-s+i+1) \lambda\left[(t+y-\mu)^{2}-\left(t+y_{r}-\mu\right)^{2}\right]}(t+y-\mu) \\
& \pi\left(\lambda, \mu \mid y_{1}, \ldots, y_{r}\right) d \lambda d \mu
\end{aligned}
$$

The above form of the posterior predictive density function is not attractable and the predictive Bayes estimate cannot be obtained in an explicit form, the Bayes Predictor (BP) of $y_{s}$ under SEL function is: 


$$
\begin{aligned}
& Y_{s: n}^{B P}=2 c \int_{y_{r}}^{\infty} y \int_{0}^{\infty} \int_{0}^{\infty} \lambda \sum_{i=0}^{s-r-1}\left(\begin{array}{c}
s-r-1 \\
i
\end{array}\right)(-1)^{i} e^{-(n-s+i+1) \lambda\left[(t+y-\mu)^{2}-\left(t+y_{r}-\mu\right)^{2}\right]}(t+y-\mu) \\
& \pi\left(\lambda, \mu \mid y_{1}, \ldots, y_{r}\right) d \lambda d \mu d y
\end{aligned}
$$

Based on MC samples $\left\{\left(\lambda_{i}, \mu_{i}\right) ; i=1,2, \ldots M\right\}$ obtained using the Gibbs sampling method, the simulation Bayes predictor is obtained to be:

$$
\begin{aligned}
& \hat{Y}_{s: n}^{B P}=\frac{2 c}{M} \sum_{j=1}^{M} \lambda_{j} \sum_{i=0}^{s-r-1}\left(\begin{array}{c}
s-r-1 \\
i
\end{array}\right)(-1)^{i} \\
& \left(\frac{e^{(n-s+i+1) \lambda_{j}\left(t+y_{r}-\mu_{j}\right)^{2}} \Gamma\left(3 / 2,(n-s+i+1) \lambda_{j}\left(t+y_{r}-\mu_{j}\right)^{2}\right)}{2\left[(n-s+i+1) \lambda_{j}\right]^{\frac{3}{2}}}+\frac{\mu-t}{2(n-s+i+1) \lambda_{j}}\right)
\end{aligned}
$$

Another important problem is to construct a two sided predictive interval of the order statistics $y_{s}$. Therefore, we need to obtain the predictive survival function of $y_{s}$ which is defined as:

$$
\begin{aligned}
& S_{y_{s} \mid y_{1}, \ldots, y_{r}}(y \mid \lambda, \mu)=\operatorname{Pr}(Y>y)=\int_{y}^{\infty} g_{y_{s} \mid y_{1}, \ldots, y_{r}}(z \mid \lambda, \mu) d z \\
& =c \sum_{i=0}^{s-r-1}\left(\begin{array}{c}
s-r-1 \\
i
\end{array}\right) \frac{(-1)^{i}}{(n-s+i+1)} e^{(n-s+i+1) \lambda\left[\left(t+y_{r}-\mu\right)^{2}-(t+y-\mu)^{2}\right]}
\end{aligned}
$$

Under the SEL function, the predictive survival function of $y_{s}$ is:

$$
\begin{aligned}
& S_{y_{s} \mid y_{1}, \ldots, y_{r}}^{P}(y \mid \lambda, \mu) \\
& =\int_{0}^{\infty} \int_{0}^{\infty} c \sum_{i=0}^{s-r-1}\left(\begin{array}{c}
s-r-1 \\
i
\end{array}\right) \frac{(-1)^{i}}{(n-s+i+1)} e^{(n-s+i+1) \lambda\left[\left(t+y_{r}-\mu\right)^{2}-(t+y-\mu)^{2}\right]} \\
& \pi\left(\lambda, \mu \mid y_{1}, \ldots, y_{r}\right) d \lambda d \mu
\end{aligned}
$$

Using the MC samples $\left\{\left(\lambda_{i} ; \mu_{i}\right) ; i=1,2, \ldots M\right\}$, the simulated estimator for the predictive survival function is given by:

$$
\begin{aligned}
& \hat{S}_{y_{s} \mid y_{1}, \ldots, y_{r}}^{P}(y \mid \lambda, \mu) \\
& =\frac{c}{M} \sum_{j=1}^{M}\left[\sum_{i=0}^{s-r-1}\left(\begin{array}{c}
s-r-1 \\
i
\end{array}\right) \frac{(-1)^{i}}{(n-s+i+1)} e^{(n-s+i+1) \lambda_{j}\left[\left(t+y_{r}-\mu_{j}\right)^{2}-\left(t+y-\mu_{j}\right)^{2}\right]}\right]
\end{aligned}
$$

Now, the (1- $\beta$ ) $100 \%$ predictive interval of $y_{s}$ can be found by solving the following two non-linear equations for the lower bound $(L)$ and upper bound $(U)$ using a suitable numerical technique. Particulary:

$$
\hat{S}_{Y_{s} \mid y_{1}, \ldots, y_{r}}^{P}(L)=1-\frac{\beta}{2} \text { and } \hat{S}_{Y_{s} \mid y_{1}, \ldots, y_{r}}^{P}(U)=\frac{\beta}{2}
$$

\section{Simulations and Data Analysis}

To illustrate the above procedures, we present the analysis of two data sets. The first data set is artificial and the second is real. The computations are performed using Mathematica 7, while the graphs are performed using Minitab. These procedures can be applied easily for any data set.

\section{Simulations}

Based on type II censored data, we report some numerical experiments performed to evaluate the behavior of the proposed methods for different sampling schemes and different priors. We have assumed $\mu=1, \lambda$ $=1.5$ to generate the Rayleigh residual life data at $t=$ 1.5. To compute the BEs under SEL function, we have assumed $\pi_{2}(\mu)$, the prior of $\mu$, has gamma density function with shape and scale parameters $c$ and $d$, respectively. To study the sensitivity of the variation in the specification of prior parameters on our Bayesian analysis, further MCMC simulations are performed using proper (informative) and improper (noninformative) priors. The hyper-parameters values of $\lambda$ and $\mu$ are $a=b=c=d=0$, we call this prior as Prior 0 . The proper priors on $\lambda$ and $\mu$ are chosen such that the prior mean of $\lambda$ is equal to 1 and its standard deviation is equal to 1 and that for $\mu$, the prior mean is 2 with a standard deviation 1.414. The corresponding to these values are $a=b=1, c=2, d=1$ (call it Prior 1). For the second proper prior (Prior 2), we assume that large amount of prior information is available and assign integer values to $\mathrm{a}, \mathrm{b}, \mathrm{c}$ and $\mathrm{d}$. That is, we assume that $a$ $=4, b=2, c=2$ and $d=4$ with prior means $2,0.5$ and prior standard deviations $1,0.354$ for $\lambda$ and $\mu$, respectively. In the third prior (Prior 3 ), we assume that another large amount of prior of information are available. The corresponding values of Prior 3 are $a=4$, $b=3, c=1$ and $d=5$. Prior 3 is more informative because it has smaller standard deviations than other 
informative priors. Specifically, the prior means of $\lambda$ and $\mu$ are equal to 1.33 and 0.2 and its standard deviations are equal to 0.67 and 0.2 .

In each sampling scheme, we compute the MLEs and the BEs for $\lambda$ and $\mu$ under SEL function and $95 \%$ credible intervals based on 10,000 MC samples. We also report the average Bayes estimates, Mean Squared Errors (MSEs) and the coverage percentage lengths based on 10,000 replications. As shown in Table 1, the MLEs and Bayes estimates of $\lambda$ and $\mu$ improve once more information is available ( $\mathrm{r}$ gets large). This is valid for all priors suggested above. The BEs or the sample-based estimates are better than the MLEs of $\lambda$ and $\mu$ (see Table 1) for all priors. If we compare between the BEs under Prior 0 and other priors, the BEs results for $\lambda$ and $\mu$ using proper priors (informative priors) are much better than the Bayes estimates of $\lambda$ and $\mu$ using Prior 0 (non-informative prior) according to the MSEs and the best prior is Prior 3 based on its
MSEs results. As shown in Table 2, we notice that the average credible intervals lengths for $\lambda$ and $\mu$ become smaller when $\mathrm{r}$ increases and $\mathrm{n}$ is fixed. It is also noticed that the average credible intervals lengths for both $\lambda$ and $\mu$ under proper priors are smaller than that under improper prior.

In the computations of predicted values, we consider both improper and proper priors under SEL function. Based on type II censored data, we have obtained the point predictors and the $95 \%$ Predictive Intervals (PIs) for the missing order statistics $Y_{s}, r<s$ $\leq n$. Based on the posterior density based method, we simulate the Bayes predictors for the missing order statistics $Y_{s}, r<s \leq n$, based on MC samples $\left\{\left(\lambda_{i}, \mu_{i}\right)\right.$; $i=1,2, \ldots, M\}$ and $M=10,000$. As shown in Table 3, we notice that the predicted values for the missing order statistics $Y_{s}$ are quite close to each other and the PIs corresponding to all four priors include the predicted values of the missing order statistics $Y_{s}$.

Table 1. MLEs and Bayes estimates with respect to SEL function based on type II censored data, when Priors $0,1,2$ and 3 are used

\begin{tabular}{|c|c|c|c|c|c|c|c|}
\hline \multirow[b]{2}{*}{$n$} & \multirow[b]{2}{*}{$r$} & \multirow[b]{2}{*}{ Parameters } & \multicolumn{5}{|l|}{ Bayes } \\
\hline & & & MLEs (MSE) & Prior 0 (MSE) & Prior 1 (MSE) & Prior 2 (MSE) & Prior 3 (MSE) \\
\hline \multirow[t]{2}{*}{25} & 10 & $\lambda$ & $1.3455(0.9596)$ & $1.3427(0.8579)$ & $1.3276(0.2500)$ & $1.32558(0.2347)$ & $1.1586(0.2266)$ \\
\hline & & $\mu$ & $0.8000(0.1406)$ & $0.9369(0.0878)$ & $0.9344(0.0690)$ & $0.8291(0.0686)$ & $0.7035(0.0197)$ \\
\hline \multirow[t]{2}{*}{25} & 15 & $\lambda$ & $1.4332(0.9491)$ & $1.3929(0.7070)$ & $1.3428(0.2290)$ & $1.3894(0.2025)$ & $1.1658(0.2061)$ \\
\hline & & $\mu$ & $0.8898(0.0541)$ & $0.9429(0.0400)$ & $0.9409(0.0354)$ & $0.8327(0.0194)$ & $0.6959(0.0192)$ \\
\hline \multirow[t]{2}{*}{25} & 20 & $\lambda$ & $1.5055(0.5097)$ & $1.5998(0.0554)$ & $1.4085(0.2184)$ & $1.4812(0.1884)$ & $1.1995(0.1740)$ \\
\hline & & $\mu$ & $0.8981(0.0293)$ & $0.9505(0.0123)$ & $0.9461(0.0115)$ & $0.8617(0.0109)$ & $0.7321(0.010)$ \\
\hline \multirow[t]{2}{*}{40} & 10 & $\lambda$ & $1.3436(0.8939)$ & $1.3936(0.8502)$ & $1.3558(0.2699)$ & $1.4298(0.2373)$ & $1.1147(0.2252)$ \\
\hline & & $\mu$ & $0.8878(0.0882)$ & $0.9210(0.0869)$ & $0.9454(0.0610)$ & $0.8356(0.0576)$ & $0.6823(0.0278)$ \\
\hline \multirow[t]{2}{*}{40} & 20 & $\lambda$ & $1.3836(0.8801)$ & $1.4324(0.7379)$ & $1.3610(0.2293)$ & $1.4533(0.2097)$ & $1.1280(0.1600)$ \\
\hline & & $\mu$ & $1.1106(0.0810)$ & $0.9542(0.0769)$ & $0.9464(0.0664)$ & $0.8463(0.0493)$ & $0.6931(0.0196)$ \\
\hline \multirow[t]{2}{*}{40} & 30 & $\lambda$ & $1.4443(0.2162)$ & $1.4942(0.1892)$ & $1.4168(0.1299)$ & $1.4707(0.1191)$ & $1.1596(0.1111)$ \\
\hline & & $\mu$ & $1.0762(0.0311)$ & $0.9590(0.0294)$ & $0.9570(0.0275)$ & $0.8936(0.0187)$ & $0.7209(0.0037)$ \\
\hline
\end{tabular}

Note: The first entry represents the point estimate and the corresponding MSE is given between parentheses.

Table 2.Average lengths credible intervals based on type II censored data and the coverage percentages, when Priors 0 , 1,2 and 3 are used

\begin{tabular}{|c|c|c|c|c|c|c|}
\hline \multirow[b]{2}{*}{$n$} & \multirow[b]{2}{*}{$m$} & \multirow[b]{2}{*}{ Parameters } & \multicolumn{4}{|c|}{ Average length of credible interval (coverage percentage) } \\
\hline & & & Prior 0 & Prior 1 & Prior 2 & Prior 3 \\
\hline \multirow[t]{2}{*}{25} & 10 & $\lambda$ & $3.2542(0.95)$ & $2.8754(0.95)$ & $2.8657(0.95)$ & $2.8589(0.94)$ \\
\hline & & $\mu$ & $0.8893(0.94)$ & $0.8475(0.95)$ & $0.8355(0.95)$ & $0.8315(0.94)$ \\
\hline \multirow[t]{2}{*}{25} & 15 & $\lambda$ & $3.1785(0.94)$ & $2.7255(0.94)$ & $2.7344(0.94)$ & $2.7019(0.95)$ \\
\hline & & $\mu$ & $0.8945(0.95)$ & $0.8335(0.96)$ & $0.8298(0.95)$ & $0.8136(0.95)$ \\
\hline \multirow[t]{2}{*}{25} & 20 & $\lambda$ & $2.9912(0.94)$ & $2.5574(0.94)$ & $2.5234(0.96)$ & $2.5036(0.96)$ \\
\hline & & $\mu$ & $0.8764(0.93)$ & $0.8225(0.94)$ & $0.8178(0.95)$ & $0.8099(0.95)$ \\
\hline \multirow[t]{2}{*}{40} & 10 & $\lambda$ & $3.2544(0.95)$ & $2.8663(0.95)$ & $2.8247(0.95)$ & $2.7692(0.95)$ \\
\hline & & $\mu$ & $0.9263(0.95)$ & $0.8278(0.95)$ & $0.8274(0.94)$ & $0.8146(0.94)$ \\
\hline \multirow[t]{2}{*}{40} & 20 & $\lambda$ & $3.0587(0.93)$ & $2.7456(0.96)$ & $2.7262(0.94)$ & $2.6938(0.95)$ \\
\hline & & $\mu$ & $0.8944(0.96)$ & $0.8044(0.96)$ & $0.8038(0.95)$ & $0.7988(0.94)$ \\
\hline \multirow[t]{2}{*}{40} & 30 & $\lambda$ & $2.9542(0.94)$ & $2.5485(0.95)$ & $2.5457(0.96)$ & $2.5134(0.96)$ \\
\hline & & $\mu$ & $0.8756(0.97)$ & $0.8001(0.96)$ & $0.7987(0.96)$ & $0.7834(0.95)$ \\
\hline
\end{tabular}


Table 3. Point predictors and PIs for the missing order statistics $\mathrm{Y}_{\mathrm{s}: n}, r<s \leq n$ Predicted value (MSE) (95\% PIs)

\begin{tabular}{|c|c|c|c|c|c|c|}
\hline \multirow[b]{2}{*}{$\mathrm{n}$} & \multirow[b]{2}{*}{$\mathrm{r}$} & \multirow[b]{2}{*}{$Y_{s: n}$} & \\
\hline & & & Prior 0 & Prior 1 & Prior 2 & Prior 3 \\
\hline \multirow[t]{8}{*}{25} & \multirow[t]{8}{*}{10} & \multirow[t]{2}{*}{$\mathrm{Y}_{11: 25}$} & $0.6315(0.0117)$ & $0.4482(0.0088)$ & $0.4599(0.0080)$ & $0.4271(0.0045)$ \\
\hline & & & $(0.5702 ; 0.8122)$ & $(0.4075 ; 0.5638)$ & $(0.4242 ; 0.5608)$ & $(0.3891 ; 0.5366)$ \\
\hline & & \multirow{2}{*}{$\mathrm{Y}_{15: 25}$} & $0.8605(0.0211)$ & $0.6236(0.0126)$ & $0.6087(0.0116)$ & $0.6053(0.0066)$ \\
\hline & & & $(0.6543 ; 1.2695)$ & $(0.4742 ; 0.8733)$ & $(0.4816 ; 0.8197)$ & $(0.4816 ; 0.8197)$ \\
\hline & & \multirow{2}{*}{$Y_{20: 25}$} & $0.9122(0.0460)$ & $0.9122(0.0204)$ & $0.8537(0.0196)$ & $0.8821(0.0119)$ \\
\hline & & & $(0.8655 ; 1.9539)$ & $(0.6312 ; 1.3742)$ & $(0.6220 ; 1.2092)$ & $(0.6123 ; 1.3132)$ \\
\hline & & \multirow[t]{2}{*}{$\mathrm{Y}_{25: 25}$} & $2.1689(0.2181)$ & $1.6022(0.0235)$ & $1.4940(0.0214)$ & $1.5903(0.0178)$ \\
\hline & & & $(1.2908 ; 3.6919)$ & $(0.97528 ; 2.6100)$ & $(0.9678 ; 1.7144)$ & $(1.1740 ; 1.7890)$ \\
\hline \multirow[t]{8}{*}{25} & \multirow[t]{8}{*}{15} & \multirow[t]{2}{*}{$Y_{16: 25}$} & $0.7765(0.0104)$ & $0.6771(0.0106)$ & $0.5957(0.0050)$ & $0.6456(0.0050)$ \\
\hline & & & $(0.7155 ; 0.9504)$ & $(0.6290 ; 0.8145)$ & $(0.5540 ; 0.7144)$ & $(0.5966 ; 0.7835)$ \\
\hline & & \multirow[t]{2}{*}{$Y_{19: 25}$} & $0.9760(0.0139)$ & $0.8401(0.0143)$ & $0.7386(0.0071)$ & $0.8204(0.0069)$ \\
\hline & & & $(0.7806 ; 1.3223)$ & $(0.6849 ; 1.1043)$ & $(0.6015 ; 0.9843)$ & $(0.6554 ; 1.1060)$ \\
\hline & & \multirow[t]{2}{*}{$\mathrm{Y}_{22: 25}$} & $1.3078(0.0228)$ & $1.0556(0.0145)$ & $0.9304(0.0111)$ & $1.0669(0.0101)$ \\
\hline & & & $(0.9343 ; 1.2562)$ & $(0.7951 ; 1.4678)$ & $(0.7016 ; 1.2802)$ & $(0.7731 ; 1.2497)$ \\
\hline & & \multirow[t]{2}{*}{$Y_{25: 25}$} & $1.9071(0.0636)$ & $1.5763(0.0531)$ & $1.4410(0.0280)$ & $1.6612(0.0230)$ \\
\hline & & & $(1.2258 ; 1.9720)$ & $(1.0451 ; 2.3988)$ & $(0.9300 ; 2.2510)$ & $(1.0680 ; 2.1164)$ \\
\hline \multirow[t]{6}{*}{25} & \multirow[t]{6}{*}{20} & \multirow[t]{2}{*}{$Y_{21: 25}$} & $1.1486(0.0196)$ & $0.8588(0.0093)$ & $0.9345(0.0064)$ & $0.9179(0.0043)$ \\
\hline & & & $(1.0456 ; 1.4337)$ & $(0.7918 ; 1.0420)$ & $(0.8710 ; 1.1085)$ & $(0.8729 ; 1.1133)$ \\
\hline & & \multirow{2}{*}{$\mathrm{Y}_{23: 25}$} & $1.3848(0.0246)$ & $1.0411(0.0126)$ & $1.1182(0.0117)$ & $1.1239(0.0107)$ \\
\hline & & & $(1.1131 ; 1.8940)$ & $(0.8421 ; 1.3998)$ & $(0.9214 ; 1.4667)$ & $(0.8986 ; 1.2326)$ \\
\hline & & \multirow{2}{*}{$Y_{25: 25}$} & $1.9544(0.0613)$ & $1.4037(0.0246)$ & $1.5166(0.0235)$ & $1.5369(0.0201)$ \\
\hline & & & $(1.3199 ; 3.0278)$ & $(0.9804 ; 2.1134)$ & $(1.0736 ; 2.2390)$ & $(1.0563 ; 2.1469)$ \\
\hline \multirow[t]{8}{*}{40} & 10 & $Y_{11: 40}$ & $0.3841(0.0073)$ & $0.3248(0.0045)$ & $0.2917(0.0028)$ & $0.3028(0.0007)$ \\
\hline & & & $(0.3502 ; 0.4833)$ & $(0.2968 ; 0.4041)$ & $(0.2699 ; 0.3546)$ & $(0.2787 ; 0.3624)$ \\
\hline & & $\mathrm{Y}_{20: 40}$ & $0.7232(0.0212)$ & $0.5663(0.0109)$ & $0.4962(0.0064)$ & $0.5369(0.0016)$ \\
\hline & & & $(0.5054 ; 1.0886)$ & $(0.4124 ; 0.4910)$ & $(0.3684 ; 0.6904)$ & $(0.3970 ; 0.6443)$ \\
\hline & & $Y_{30: 40}$ & $1.1519(0.0480)$ & $0.8361(0.0117)$ & $0.7651(0.0109)$ & $0.8826(0.0086)$ \\
\hline & & & $(0.7729 ; 0.7561)$ & $(0.5584 ; 0.1804)$ & $(0.5462 ; 1.0734)$ & $(0.6011 ; 0.8854)$ \\
\hline & & $Y_{40: 40}$ & $2.4033(0.4506)$ & $1.7479(0.0318)$ & $1.5421(0.0280)$ & $1.8414(0.0253)$ \\
\hline & & & $(1.3851 ; 2.5279)$ & $(1.0522 ; 2.1124)$ & $(0.9706 ; 2.3846)$ & $(1.1526 ; 2.1206)$ \\
\hline 40 & 20 & $Y_{21: 40}$ & $0.6716(0.0099)$ & $0.5101(0.0024)$ & $0.5322(0.0022)$ & $0.5107(0.0015)$ \\
\hline & & & $(0.6373 ; 0.7687)$ & $(0.4850 ; 0.5806)$ & $(0.5072 ; 0.6033)$ & $(0.4846 ; 0.5738)$ \\
\hline & & $Y_{28: 40}$ & $0.8236(0.0129)$ & $0.6154(0.0028)$ & $0.6377(0.0028)$ & $0.6272(0.0025)$ \\
\hline & & & $(0.6926 ; 1.040)$ & $(0.5132 ; 0.7282)$ & $(0.5476 ; 0.7797)$ & $(0.5487 ; 0.7693)$ \\
\hline & & $Y_{35: 40}$ & $1.3485(0.0298)$ & $1.0258(0.0146)$ & $1.0066(0.0117)$ & $1.0585(0.0094)$ \\
\hline & & & $(1.0010 ; 1.8677)$ & $(0.7638 ; 1.4080)$ & $(0.7673 ; 1.3371)$ & $(0.7881 ; 1.3412)$ \\
\hline & & $\mathrm{Y}_{40: 40}$ & $2.1214(0.1077)$ & $1.5873(0.0198)$ & $1.6504(0.0146)$ & $1.7337(0.0113)$ \\
\hline & & & $(1.3861 ; 3.2558)$ & $(1.0632 ; 1.8340)$ & $(1.1017 ; 2.5178)$ & $(1.1341 ; 2.1837)$ \\
\hline 40 & 30 & $Y_{31: 40}$ & $1.0139(0.0077)$ & $0.8308(0.0072)$ & $0.7774(0.0066)$ & $0.8041(0.0043)$ \\
\hline & & & $(0.9624 ; 1.1577)$ & $(0.7935 ; 0.9331)$ & $(0.7434 ; 0.8723)$ & $(0.7634 ; 0.8767)$ \\
\hline & & $Y_{34: 40}$ & $1.1793(0.0086)$ & $0.9626(0.0082)$ & $0.9044(0.0074)$ & $0.9515(0.0059)$ \\
\hline & & & $(1.0184 ; 1.4607)$ & $(0.8382 ; 1.1745)$ & $(0.7868 ; 1.0973)$ & $(0.8134 ; 1.0837)$ \\
\hline & & $Y_{37: 40}$ & $1.4424(0.0122)$ & $1.1655(0.0115)$ & $1.1020(0.0103)$ & $1.1591(0.0089)$ \\
\hline & & & $(1.1438 ; 1.9142)$ & $(0.9383 ; 1.5020)$ & $(0.8847 ; 1.4317)$ & $(0.9199 ; 1.4163)$ \\
\hline & & $\mathrm{Y}_{40: 40}$ & $2.0298(0.0507)$ & $1.6314(0.0120)$ & $1.5004(0.0114)$ & $1.6897(0.0110)$ \\
\hline & & & $(1.4286 ; 2.9836)$ & $(1.1660 ; 2.3308)$ & $(1.0668 ; 2.2059)$ & $(1.1545 ; 2.2358)$ \\
\hline
\end{tabular}

Note: The first entry represents the point predictor, the corresponding MSE is given between parentheses and the corresponding predictive interval is given below between parentheses.

It is also noticed that the MSEs of the predicted values increase when the values of $r$ become large. This means that the prediction worsen when we predict values that are much more far from the observed values. Hence, the prediction of the closer values to the observed ones is much accurate than the prediction of most wider values. In terms of MSEs, the Bayes predictors under prior 3 work better than the Bayes predictor under priors 1 and 2. In fact, the MSEs of the Bayes predictors decrease as the standard deviation of the proper prior decreases and since Prior 3 is more informative (i.e., has minimum standard deviation) it has the least MSEs results over the other priors. It can also be seen from Table 3 that our 
results are not very sensitive to the assumed values of the proper prior parameters.

\section{Data Analysis}

In this subsection we analyze the ball bearings data which were originally reported by Lawless (1982, p. 288 ) and represent the number of million revolutions before failing for each of 25 ball bearings in a life test. These data were studied by several authors; Gupta and Kundu (2001) used these data set to compare gamma, Weibull and generalized exponential models. They compare between the three distributions in the estimation not prediction point of view. Meintanis (2008) showed that the generalized Rayleigh distribution works quite well for these ball bearing data. Raqab and Madi (2011) used these data to estimate the two parameters of the generalized Rayleigh distribution and to predict the censored values of these data under the type II progressively censoring scheme. The prediction results performed using the method presented in this study are better than the results presented in Raqab and Madi (2011), this could be noticed if anyone compares between the PIs lengths of the two papers. The predicted values in the two papers are very close to each other. Raqab and Madi (2011) did not include their MSEs results but our MSEs results are very small which means that our prediction results are very accurate.

The type II censored sample we use is as follows: $\left\{X_{i}, i=1, \ldots, 25\right\}=\{17.88,28.92,33.00,41.52,42.12$, 45.60, 48.48, 51.84, 51.96, 54.12, 55.56, 67.80, 67.80, $67.80,68.64,86.64,68.88,84.12,93.12,98.64\}$. The sample consists of the smallest twenty lifetimes out of twenty five failure times of ball bearings in endurance test. In this example we propose that the predetermined time $t$ is 1.5 to denote that the ball bearing that fails before 1.5 million revolutions has an artificial error. All lifetimes that are less than $t$ will be deleted, in this example we consider all lifetimes because each of them is greater than $t$. As presented in the previous section, our interest is in the random variable $Y=X$-t . The maximum likelihood estimates based on the above data were determined to be $\hat{\mu}=$ $0.7572, \hat{\lambda}=0.0197$. The Bayes estimates using Prior 1 parameter values turned out to be $\hat{\mu}_{\text {Bayes }}^{P 1}=0.9911$, $\hat{\lambda}_{\text {Bayes }}^{P 1}=0.0223$ while the Bayes estimates using Prior 3 parameter values turned out to be $\hat{\mu}_{\text {Bayes }}^{P 1}=0.8947$, $\hat{\lambda}_{\text {Bayes }}^{P 1}=0.0215$. Further, the $95 \%$ prediction intervals for $\mu$ and $\lambda$ under Prior 1 are determined to be $(0.5620 ; 1.4490)$ and $(0.0138 ; 0.0320)$ while the $95 \%$ prediction intervals for $\mu$ and $\lambda$ under Prior 3 are determined to be $(0.6021 ; 1.3972)$ and $(0.0168$; $0.0301)$, respectively. The Bayes point predictors and the $95 \%$ prediction intervals for the missing order statistics $\left\{X_{s: n}, 21 \leq s \leq 25\right\}$ after re-parametrization, when Priors 1 and 3 are used, are presented in Table 5 below.

It is observed that all predicted values with respect to SEL function are ordered and fall in their corresponding PIs. As shown in Table 5, the MSEs results and the PIs lengths for Prior 3 are better than that of Prior 1 because Prior 3 is more informative than Prior 1. Further, the kernel method is used to present the plots of the predictive densities of $Y_{21}, Y_{22}$, $Y_{23}, Y_{24}$ and $Y_{25}$ in Figure 1-5.

Table 4. MLEs and Bayes estimates for the real data.

\begin{tabular}{llllll}
\hline $\mathrm{n}$ & $\mathrm{m}$ & Parameters & MLEs (MSE) & Bayes (Prior 1) (MSE) & Bayes (Prior 3) (MSE) \\
\hline 25 & 20 & $\lambda$ & $0.0197(0.0036)$ & $0.0223(0.0033)$ & $0.0215(0.0028)$ \\
& $\mu$ & $0.7572(0.0799)$ & $0.9911(0.0686)$ & $0.8947(0.0539)$ \\
\hline
\end{tabular}

Table 5. Point predictors and PIs for the missing order statistics $Y_{s: n}, r<s \leq n$

Predicted value (MSE) (95\% PIs)

\begin{tabular}{|c|c|c|c|c|}
\hline \multirow[b]{2}{*}{$\underline{n}$} & \multirow[b]{2}{*}{$r$} & \multirow[b]{2}{*}{$Y_{s: n}$} & \\
\hline & & & Prior 1 & Prior 3 \\
\hline \multirow[t]{10}{*}{25} & 20 & $Y_{21: 25}$ & $104.468(0.0010)$ & $103.541(0.0008)$ \\
\hline & & & $(95.788 ; 120.050)$ & $(98.765 ; 116.620)$ \\
\hline & & $Y_{22: 25}$ & $111.228(0.0013)$ & $109.436(0.0012)$ \\
\hline & & & $(100.176 ; 133.600)$ & $(99.959 ; 128.590)$ \\
\hline & & $Y_{23: 25}$ & $120.250(0.0020)$ & $116.580(0.0017)$ \\
\hline & & & $(103.129 ; 150.670)$ & $(102.238 ; 141.600)$ \\
\hline & & $Y_{24: 25}$ & $131.700(0.0065)$ & $126.370(0.0035)$ \\
\hline & & & $(107.587 ; 172.370)$ & $(106.199 ; 159.760)$ \\
\hline & & $Y_{25: 25}$ & $151.790(0.0124)$ & $144.150(0.0078)$ \\
\hline & & & $(115.400 ; 211.200)$ & $(112.850 ; 195.740)$ \\
\hline
\end{tabular}




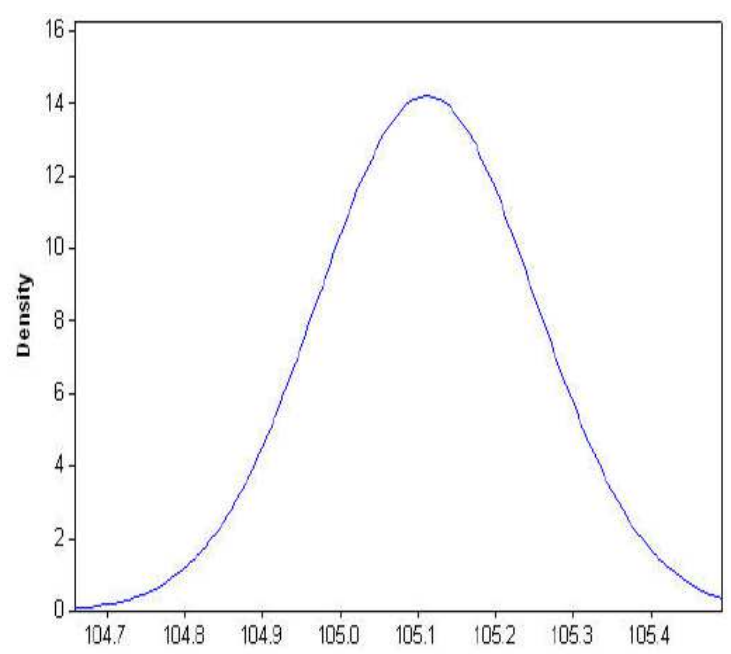

Fig. 1. Estimate of the predictive density function for $Y_{21}$

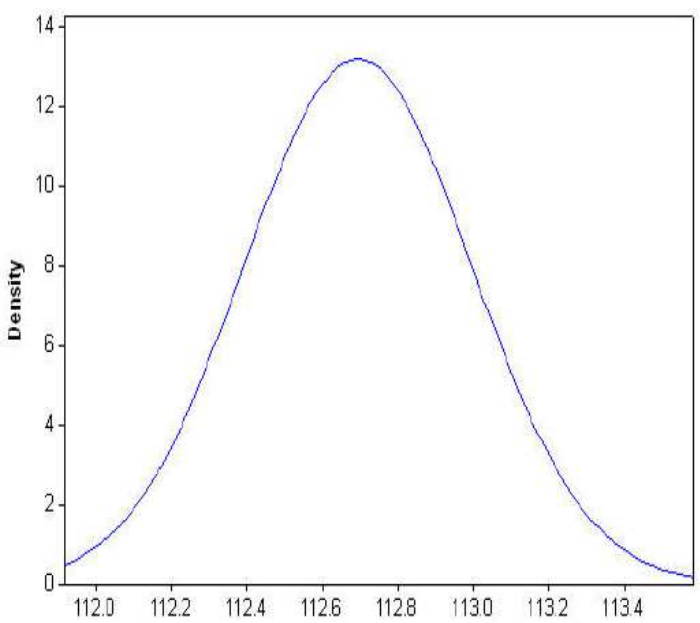

Fig. 2. Estimate of the predictive density function of $\mathrm{Y}_{22}$

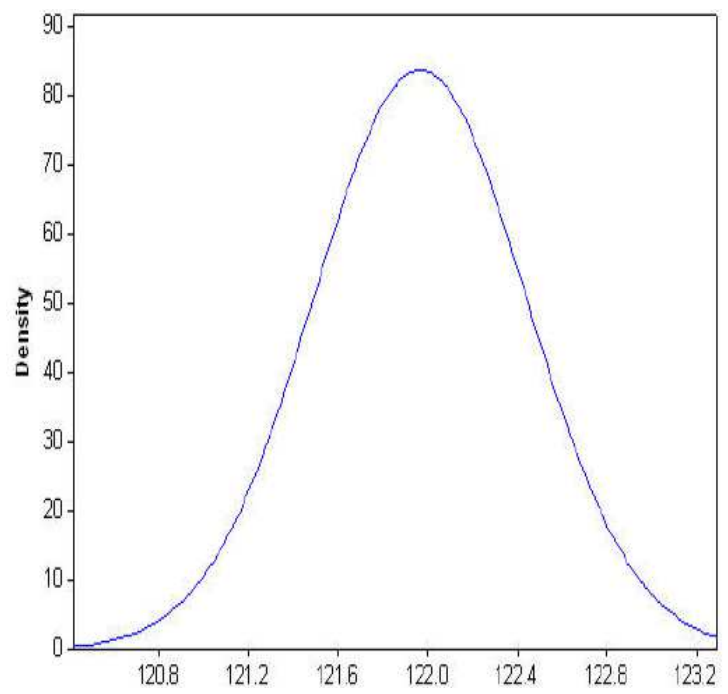

Fig. 3. Estimate of the predictive density function for $\mathrm{Y}_{23}$

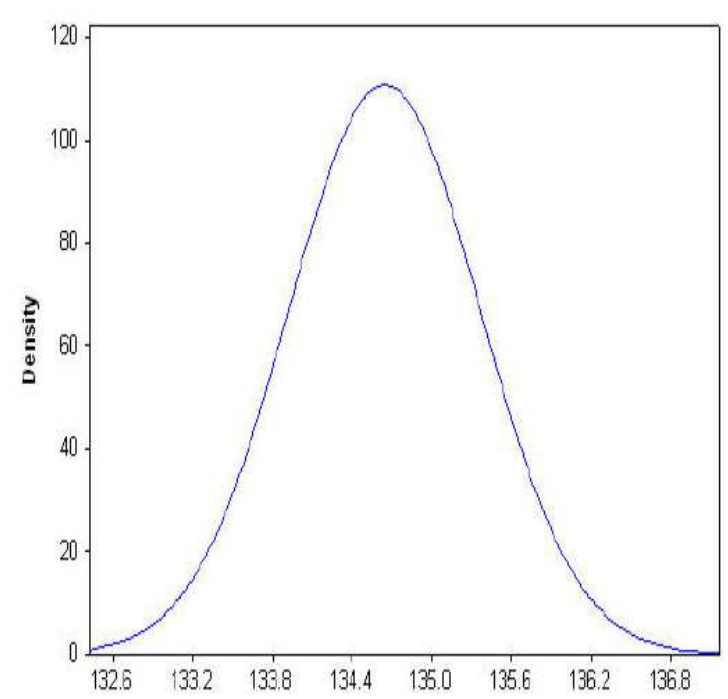

Fig. 4. Estimate of the predictive density function of $\mathrm{Y}_{24}$

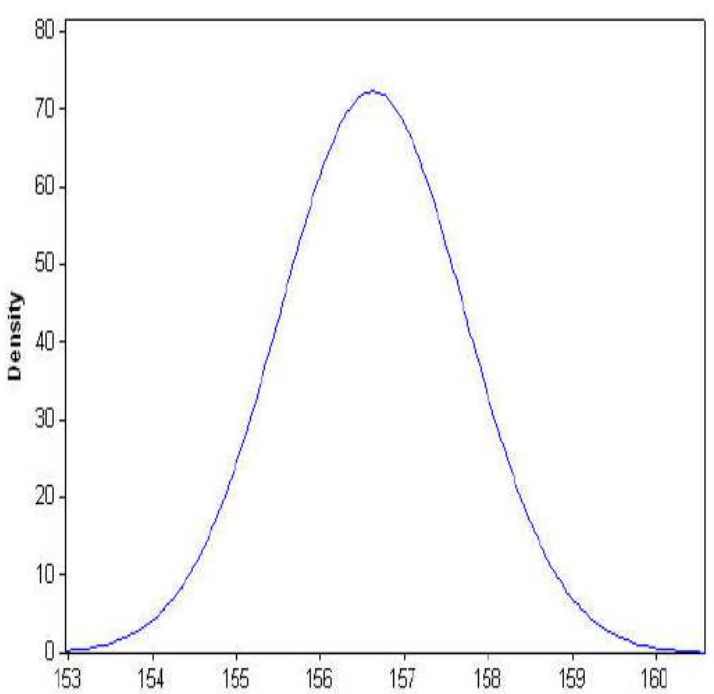

Fig. 5. Estimate of the predictive density function for $\mathrm{Y}_{25}$

\section{Conclusion}

In this article, we have studied the problem of estimation and prediction for the two-parameter Rayleigh distribution under residual type-II censored data. In the estimation problem, we have computed the maximum likelihood estimators along with the Bayes estimations for the model parameters. Based on the simulation part of the paper, we have proved that the Bayes estimation method is better than the maximum likelihood method of estimation. It is also proved that the Bayes estimation method under more informative priors is better than Bayes estimation under less informative, non-informative and the maximum likelihood estimation method. In the prediction problem, we have used the Gibbs and Metropolis samplers to predict the residual 
times to failure units, $Y_{i: n}, i=j+1,1, \ldots, n$, that remain based on the last observed time. It is proved that the predicted values under more informative priors are better than the less informative and non-informative priors. Depending on these results, we have applied the above obtained results in estimation and predicting of a real life ball bearings data.

\section{Acknowledgment}

The authors would like to thank the editor, Prof. Samaranayake, V.A. and anonymous referee for their very constructive suggestions that contributed to the improvement of this version of the paper.

\section{Author's Contributions}

All Authors have contributed equally.

\section{Ethics}

The authors declare that there is no conflict of interests regarding the publication of this paper.

\section{References}

Balakrishnan, N. and M. Kateri, 2008. On the maximum likelihood estimation of parameters of Weibull distribution based on complete and censored data. Stat. Probability Lett., 78: 2971-2975. DOI: $10.1016 /$ j.spl.2008.05.019

Balakrishnan, N. and C.R. Rao, 1997. Large-sample approximations to the best linear unbiased estimation and best linear unbiased prediction based on progressively censored samples and some applications. In: Advances in Statistical Decision Theory and Applications, Panchapakesan, S. and N. Balakrishnan (Eds.), Birkhauser, Boston, ISBN-10: 1461274958, pp: 431-444.

Chen, M.H., Q.M. Shao and J.G. Ibrahim, 2000. Monte Carlo Methods in Bayesian Computation. 1st Edn., Springer-Verlag, New York, ISBN-10: 0387989358, pp: 387.

David, H.A. and H.N. Nagaraja, 2003. Order Statistics. 3rd Edn., Wiley, New York.

Dey, S., 2009. Comparison of Bayes estimators of the par ameter and reliability function for Rayleigh distribution under different loss functions. Malaysian J. Math. Sci., 3: 247-264.

Dey, S., T. Dey and D. Kundu, 2014. Two-parameter Rayleigh distribution: Different methods of estimation. Am. J. Math. Manage. Sci., 33: 55-74.
Dey, T., S. Dey and D. Kundu, 2016. On progressively type-II censored two-parameter Rayleigh distribution. Commun. Stat. Simulat. Comput., 45: 438-455. DOI: 10.1080/03610918.2013.856921

Gupta, R.D. and D. Kundu, 2001. Exponentiated exponential family: An alternative to Gamma and Weibull distributions. Biometrical J., 43: 117-130.

Johnson, N.L., S. Kotz and N. Balakrishnan, 1994. Continuous Univariate Distributions. 2nd Edn., Wiley-Interscience, ISBN-10: 0471584959, pp: 761.

Kaminskiy, M.P. and V.V. Krivtsov, 2005. A simple proccedure for Bayesian estimation of the Weibull distribution. IEEE Trans. Reliability Anal., 54: 612616. DOI: 10.1109/TR.2005.858093

Kaminsky, K.S. and P.I. Nelson, 1998. Prediction of Order Statistics. In: Order Statistics: Applications, Balakrishnan, N. and C.R. Rao (Eds.), North-Holland, Amsterdam, ISBN-10: 0444829229, pp: 431-450.

Kundu, D., 2008. Bayesian inference and life testing plan for the Weibull distribution in presence of progressive censoring. Technometrics, 50: 144-154.

Lawless, J.F., 1982. Statistical Models and Methods for Lifetime Data. 1st Edn., Wiley, New York, ISBN-10: 0471085448, pp: 580.

Meintanis, S.G., 2008. A new approach of goodness-offit testing for exponentiated laws applied to the generalized Rayleigh distribution. Computat. Stat. Data Anal., 52: 2496-2503. DOI: $10.1016 /$ j.csda.2007.08.014

Miller, K.S. and H. Sackrowttz, 1967. Relationships between biased and unbiased rayleigh distributions. SIAM J. Applied Math., 15: 1490-1495. DOI: $10.1137 / 0115130$

Rayleigh, J.W.S., 1880. On the resultant of a large number of vibrations of the some pitch and of arbitrary phase. Philosophical Magazine, Series 5, 10: 73-78. DOI: 10.1080/14786448008626893

Raqab, M.Z. and M.T. Madi, 2011. Inference for the generalized Rayleigh distribution based on progressively censored data. J. Stat. Plann. Inference, 141: 3313-3322. DOI: $10.1016 /$ j.jspi.2011.04.016

Siddiqui, M.M., 1962. Some problems connected with Rayleigh distribution. J. Res. Nat. Bureau Standards, 66D: 167-174. DOI: 10.6028/jres.066d.020 